\title{
Inverse kinematic analysis of 3 DOF 3-PRS PM for machining on inclined prismatic surfaces
}

\author{
Hishantkumar Rashmikantbhai Patel, Yashavant Patel \\ Mechanical Engineering Department, A D Patel Institute of Technology, India
}

\section{Article Info}

Article history:

Received Sep 18, 2019

Revised Oct 06, 2019

Accepted Feb 18, 2020

Keywords:

3 PRS

Inverse kinematic

Parallel manipulator

Prismatic surfaces

\begin{abstract}
Parallel Manipulators (PMs) are family members of modern manipulators based on the closed loop structural architecture. 3-PRS (prismatic, revolute, spherical) manipulator with $3 \mathrm{DOF}$ is investigated for its machining capability on prismatic surfaces as it possesses greater structural stiffness, higher pay load caring capacity, more precision compare to serial manipulators as well as less accumulation of errors at joints within a constrained workspace. The said manipulator can be utilized in various fields of application such as precise manufacturing, medical surgery, space technology and many more. In this paper, the primary focus on usage of parallel manipulator in industrial applications such as drilling and grooving on inclined work part surface. Inverse kinematic solutions are used for drilling, square and round profiles on inclined surface using parallel manipulator.
\end{abstract}

This is an open access article under the CC BY-SA license.

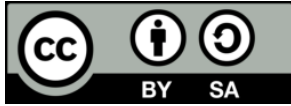

\section{Corresponding Author:}

Hishantkumar Rashmikantbhai Patel, Mechanical Engineering Department,

A D Patel Institute of Technology,

New Vallabh Vidyanagar Gujarat, India.

Email: hishupatel111@gmail.com

\section{INTRODUCTION}

In recent era, there are lot of research works going on parallel manipulators (PMs)due to their inherent qualities for precise manufacturing or precise working in smaller operating workspace. Following three elements are commonly used in any parallel mechanisms such as, 1) fixed platform, 2) connecting links and 3) moving platform. Various configurations of parallel mechanisms and their applications such as food packaging industry and automatic spray printing etc. Cable driven robots are applicable in cutting, excavating as well as grinding. 3PUU and 3-PRS parallel manipulators are used in medical and machining applications respectively as reported by Y.D Patel et al. [1].

Now days, high- speed machining of extra-large component with complex geometries is one of the challenging problem in machine tool industry. For instance, machining an aircraft component having large cross section would require a huge gantry 5-axis machine tool with tons of weight and large footprint. One auspicious alternative solution is the use of parallel kinematic mechanism in place of large machine tool. This proposal has been fully exemplified by commercial susses of Tricept robotics [2] and sprint $Z_{3}$ [3] head in locomotive and aerospace industries. HUANG, et al. proposed novel parallel kinematic machine (PKM) named A3 head which has one transitional and two rotational capabilities. Added by $\mathrm{x}-\mathrm{y}$ motion, proposed module can be used as a multiple axis spindle head to form a 5-axis high speed machining unit. Motivated by machining using conventional machine required additional support structure like jigs and fixture. One can use this parallel kinematic structure in place of conventional machine which reduce production time as well as production cost. Forward kinematic is required for synthesis of new robotics configuration. Inverse kinematic is mandatory for its real application during machining requirements. The direct kinematic analysis 
as well as work space is generated for 3DOF 3-PRS (prismatic, revolute and spherical) parallel manipulator reported by Y. D Patel et al. [4]. Y.D Patel et al. are carried out inverse kinematic analysis of 3DOF 3PRS parallel manipulator based on constraint equation [5]. Such 3 DOF 3-PRS parallel mechanism is used for generating complex geometry on inclined surfaces. Pond et al. presented variation in vertical guide way of 3 -PRS manipulator and state that Tsai et al. Model has maximum reachable workspace compare to Carretero et al. And Merlet's model [6]. Arockia Selvakumar A et al. proposed Three methods are used for position analysis of 3 DOF tripode and tri-glider. 1) Simulation in ADAMS software, 2) fabricated model and 3) analytical method [7]. Saioa Herrero et al. are used2PRU-1PRS parallel manipulator in place of 3 PRS parallel manipulator for kinematic investigation [8]. In parallel manipulator, torque at joints and angular tilt of mobile platform are key parameters for accurate position as well as orientation. Comparative study of 3UPS, 3-RPR and 3-RPS 3DOF parallel manipulators are carried out based on the torque at joints, angular tilt of moving platform and positional accuracy by A. Arockia et al. [9].

A 3-PRS parallel manipulator with adjustable layout angle of actuators is used for kinematic investigation. Yangmin Li et al. reported maximum reachable work space occurred at $75^{\circ}$ [10]. Pardeshi et al. presented parallel mechanism with less degree of freedom is more suitable for drilling, milling, welding and tapping application. 3 DOF parallel manipulators have grater advantages over high degree of freedom parallel manipulators in terms of manufacturing and operating cost. 3 DOF parallel manipulators can be classified in three categories"

Planner parallel manipulator (3 translational motion)

Spherical parallel manipulator (3 rotational motion)

Spatial parallel manipulator (Mixed motion) [11].

Tsai et al. [12] represent the geometric relation between sliders, links and moving platform are built to formulate three nonlinear trigonometric equations and this nonlinear equation are solved using Bezouts methods. Cherfia et al. [13] are carried out inverse and direct kinematic analysis of constrained parallel robot with 3 dof and four passive segments. Validate the results of direct and inverse kinematic with experimental prototype. Zhang et al. [14] carried out inverse position analysis of 3 DOF A3 head. Zhang et al. [14] state that structural parameters of 3-RPS mechanism have a slight effect on the joints motion. Zhang et al. [15] depicts the effect of the radii of the platform and the base along with the cross-section of the limb on the lower order frequency. This data is useful in the design stage.

In this paper, spatial parallel manipulator with linear actuations of lead screw are determined for drilling holes on inclined surfaces with confined workspace. 3 DOF parallel manipulator with moving platform is connected with base platform by means of links with prismatic-revolute-spherical joints. Revolute and spherical joints are passive whereas prismatic joints are active joints.

This paper deals with inverse kinematic analysis of said parallel manipulator on inclined prismatic surfaces. This work is organized in five sections in this paper. Architecture of proposed manipulator is discussed in second section. Third section deals with mobility equation as well as degree of freedom calculation for 3-PRS parallel mechanism. Inverse kinematic analysis of 3-PRS parallel manipulator is carried out in fourth section. In the fifth section, position analysis for drilling and profile generation is carried out for proposed manipulator.

\section{ARCHITECTURAL DESCRIPTION}

The schematic representation of proposed 3-DOF parallel manipulator is shown in Figure 1. Moving platform is connected to the base platform by three limbs. Each limb consists of prismatic-revolute-spherical joints. These three limbs will make manipulator symmetric. Each limb has one active prismatic joint and two passive revolute and spherical joints. All active joints are located on/near to the base platform. Three active prismatic joints have recirculating ball screw mechanism which enables us to stop prismatic motion at any instant without any slippage. Simultaneous variable motions of prismatic joints are responsible for the angular tilt of the moving platform, which is desired for drilling application. Here, $U_{1}, U_{2}$, and $U_{3}$ are lengths of connecting links 1,2 and 3 respectively. All prismatic joints makes $120^{\circ}$ with each other, if vectors are drawn from origin ' $\mathrm{O}$ ' in $\mathrm{XY}$ plane. Fixed and moving platforms are equilateral triangles in shape as shown in Figure 2.

\section{MOBILITY AND DEGREE OF FREEDOM}

Mobility analysis is carried out for many parallel manipulators using Grübler-Kutzbach criterion. Using Grübler-Kutzbach equation for 3 PRS parallel mechanism, 


$$
\begin{aligned}
& D O F=\lambda(n-j-1)+\sum_{i=1}^{j} f_{i} \\
& D O F=6(8-9-1)+(1 \times 3+1 \times 3+3 \times 3)=3
\end{aligned}
$$

here, $\lambda=$ degree of freedom of space utilized for a mechanism while in operation. For above configuration, for spatial motion $\lambda=6, n$ is a number of links, $j$ is number of joints and $f_{i}$ is degree of freedom for $i^{t h}$ joints. Hence, 3-PRS manipulator has three degrees of freedom. Grübler-Kutzbach criterion was specifically used for determining the number of degree of freedom. It cannot find what type of degree of freedom present in the manipulator. It was observed that 3 DOF manipulator has one translation motion along the $\mathrm{Z}$ axis and two rotational motions about $\mathrm{X}$ and $\mathrm{Y}$ axis.

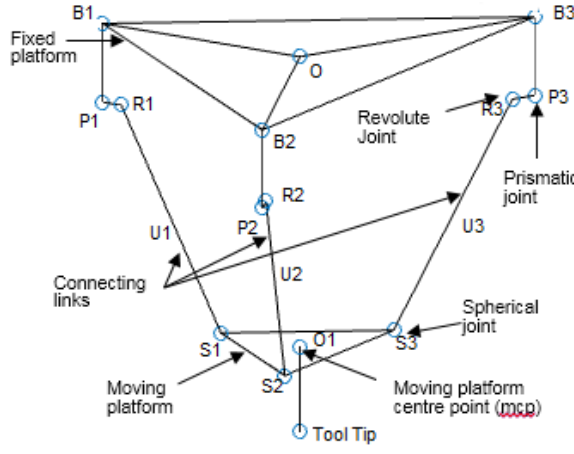

Figure 1. Schematic diagram of 3 PRS parallel mechanism

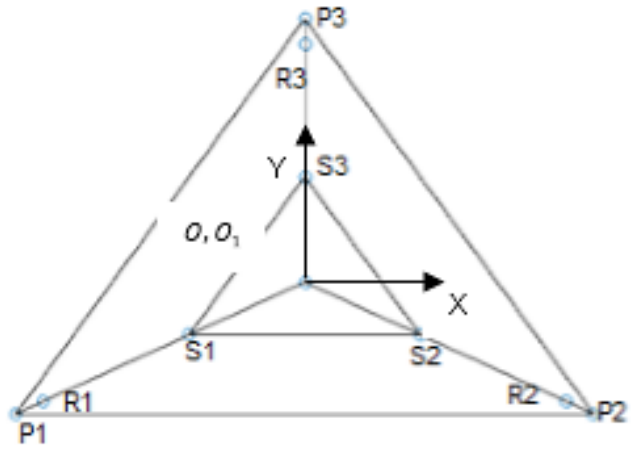

Figure 2. Planner view of 3 PRS mechanism

\section{INVERSE KINEMATIC ANALYSIS}

Inverse kinematic problem can be defined as placement of tool frame at desired position and orientation by means of actuation. The purpose of inverse kinematic problem is to determine actuated variable from a given position of end effector. Inverse kinematic analysis was used in practical application of manipulator. Figure 3, shows position vectors of spherical joints with respect to reference frame $T_{1}, T_{2}$, and $T_{3}$ are linear actuation of prismatic joints of limbs 1, 2 and 3 respectively. $p$ is a distance between two lead screw, $q$ is a centre to centre distance between to spherical joints and $\mathrm{b}$ is centre to centre distance between prismatic joint and revolute joint.

Spherical joints coordinates are expressed in the form of,

$$
n \cdot\left(S-O_{1}\right)=0
$$

$n$ is a normal vector passing through the point $O_{1}$ and centre points coordinates of any spherical joints lying in this plane with coordinates $S_{i}\left(S_{i x}, S_{i y}, S_{i z}\right)$, as shown in Figure 4.

The equation of sphere passing through three spherical joints is expressed as,

$$
\left(S_{i x}-x_{0}\right)^{2}+\left(S_{i y}-y_{0}\right)^{2}+\left(S_{i z}-z_{0}\right)^{2}-R_{m}^{2}
$$

here $i=1,2,3$ and $S_{i x}, S_{i y}$ and $S_{i z}$ are spherical joints coordinate in $\mathrm{x}, \mathrm{y}$ and $\mathrm{z}$ axis respectively.

$$
=\left[\begin{array}{ccc}
S_{1 x} & S_{2 x} & S_{3 x} \\
S_{1 y} & S_{2 y} & S_{3 y} \\
S_{1 z} & S_{2 z} & S_{3 z} \\
1 & 1 & 1
\end{array}\right]
$$

Spherical joints angles are determined by following (4),

$$
\begin{aligned}
& S_{1 x}=-\frac{q}{2}+\frac{\sqrt{3}}{2} b+\frac{\sqrt{3}}{2} U \cos \theta_{1} \\
& S_{1 y}=-\frac{q}{2 \sqrt{3}}+\frac{1}{2} b+\frac{1}{2} U \cos \theta_{1}
\end{aligned}
$$




$$
\begin{aligned}
& S_{1 z}=T_{1}+U \sin \theta_{1} \\
& S_{2 x}=\frac{q}{2}-\frac{\sqrt{3}}{2} b-\frac{\sqrt{3}}{2} U \cos \theta_{2} \\
& S_{2 y}=-\frac{q}{2 \sqrt{3}}+\frac{1}{2} b+\frac{1}{2} U \cos \theta_{2} \\
& S_{2 z}=T_{2}+U \sin \theta_{2} \\
& S_{3 x}=0 \\
& S_{3 y}=\frac{q}{\sqrt{3}}-b-U \sin \theta_{3} \\
& S_{3 z}=T_{3}+U \sin \theta_{3}
\end{aligned}
$$

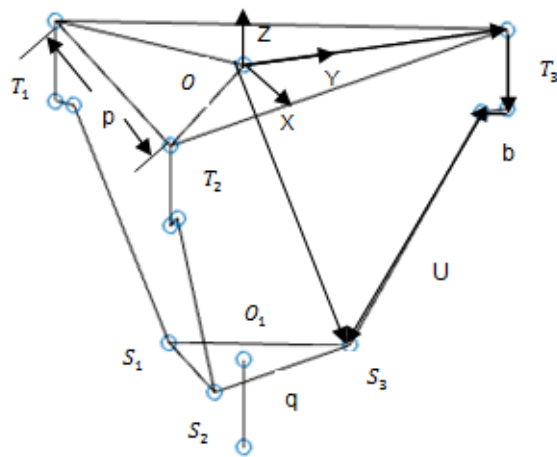

Figure 3. Position vector of spherical joint

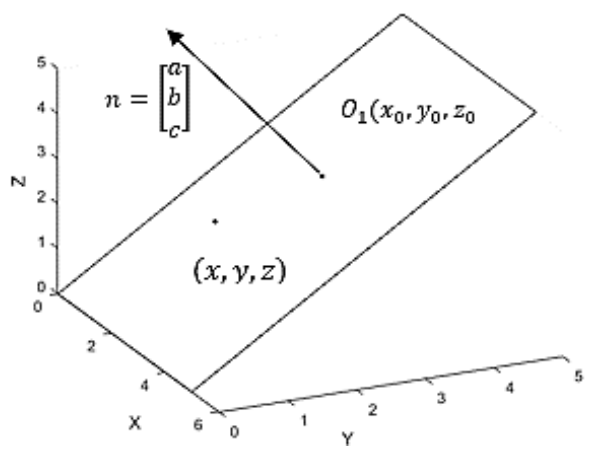

Figure 4. Normal vector passing through the plane

Case study 1: Structural parameters considered for 3PRS manipulator (Table 1 and Figure 5) are:

$$
\begin{aligned}
& \text { tool tip }=(-1.459,-2.526,-756.943) \\
& M c p=(0.0104,-0.005926,-581.967)
\end{aligned}
$$

Table 1. Joints parameters for case study 1

\begin{tabular}{cc}
\hline$\theta$ & Linear actuation \\
\hline$\theta_{1}=62.9989^{\circ}$ & Required linear actuation, $\mathrm{T}_{1}=10 \mathrm{~mm}$ \\
$\theta_{2}=63.0037^{\circ}$ & Required linear actuation, $\mathrm{T}_{2}=7.5 \mathrm{~mm}$ \\
$\theta_{3}=62.9989^{\circ}$ & Required linear actuation, $\mathrm{T}_{3}=5 \mathrm{~mm}$ \\
\hline
\end{tabular}

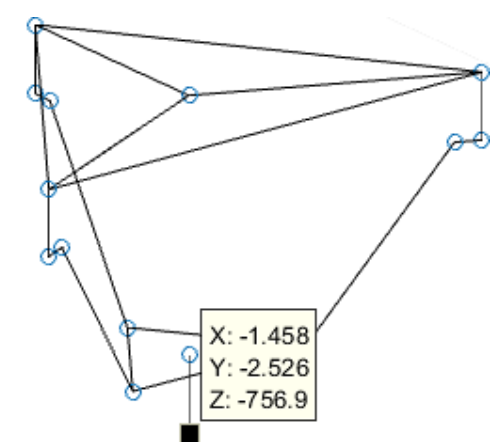

Figure 5. Tool position and orientation after actuation 


\section{POSITION ANALYSIS OF MANIPULATOR FOR DRILLING AND SLOTTING OPERATION ON PRISMATIC SURFACE}

Placement of tool frame at pre-defined point on surface is called position analysis. In which, this pre-defined point is considered as a tool tip. The tool tip coordinates are used to determine linear actuations of recirculating ball screw. Figure 6, shows prismatic part with inclined surface. Inclination angle of inclined surface $\theta$ will be varied as per requirements. Selected dimension of prismatic part was lies in the workspace of the said manipulator. Consider one new coordinate system ' $O_{2}$ ' at one bottom corner of the prismatic part. This prismatic part was placed at off-set to the 3 PRS parallel manipulator reference frame ' $O{ }^{\prime}$. The points required for a position analysis should be measure from the reference coordinate system ' $O$ '. It should be essential to find out normal vector to the inclined surface for proper orientation of the tool frame.

In Figure 7 show normal vector to the plane as well as offset distance between two coordinate systems. Determined by (4).

$$
\begin{aligned}
& \text { normal vector position }=T L\left(\frac{\overrightarrow{X_{12}} \times \overrightarrow{X_{13}}}{\left|\overrightarrow{\overline{12}_{2}} \times \overline{X_{13}}\right|}\right) \\
& \vec{X}=O\left(O_{x}, O_{y}, O_{z}\right)-O_{2}\left(O_{2 x}, O_{2 y}, O_{2 z},\right)
\end{aligned}
$$

Here,

$\overrightarrow{X_{12}}$ Is position vector between point 2 and 1

$\overrightarrow{X_{13}}$ Is position vector between point 3 and 1

TL is a tool length (vector length)

$\vec{X}$ Is offset distance between ' $O$ ' and ' $O_{1}$ '

Following steps are followed in position analysis:

1. Find out points coordinate with respect to mechanism reference frame ' $O$ '.

$$
\text { tool tip }=\text { point coordinate }\left(\overrightarrow{P_{l}}\right)-\text { offset distance }(\vec{X})
$$

2. Determine the normal vector to the plane and its end point.

$$
\text { End point }=\text { start point }-T L\left(\frac{\overrightarrow{X_{12}} \times \overrightarrow{X_{13}}}{\overrightarrow{X_{12}} \times \overline{X_{13}}}\right)
$$

3. Compute moving platform centre point (mcp)

$$
m c p=\text { end point }- \text { off set distance }(\vec{X})=\text { tool tip }-\vec{n}
$$

Position analysis of manipulator for drilling and slotting operation on inclined surface are shown in case study 2(a) and 2(b) respectively.

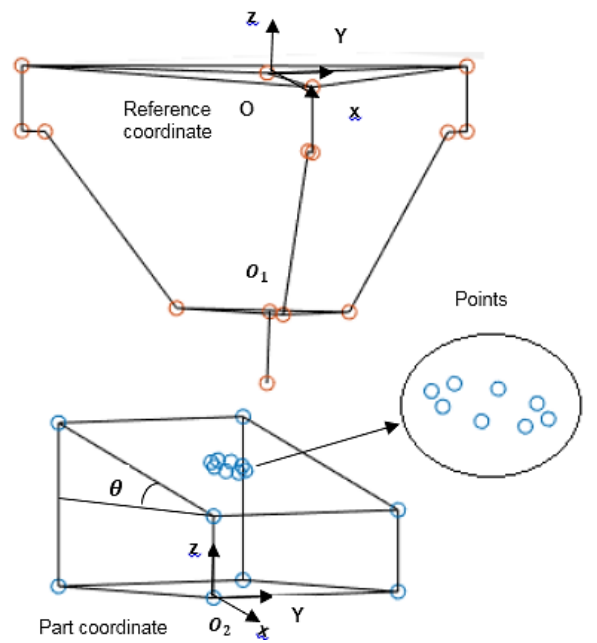

Figure 6. Number of points on prismatic parts

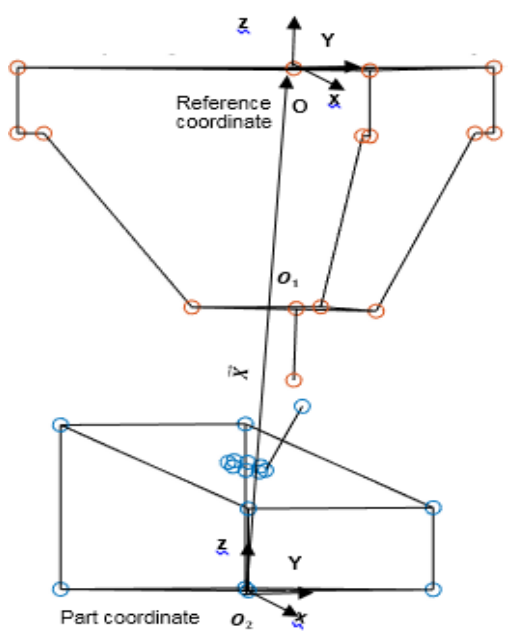

Figure 7. Normal vector and its end point on prismatic parts

Inverse kinematic analysis of 3 DOF 3-PRS PM for machining... (Hishantkumar Rashmikantbhai Patel) 


\section{Case study 2(a)}

Consider a one point $P_{1}$ on inclined prismatic surface $\theta=26.5651^{\circ}$

Offset distance, $\vec{X}=(100.0000,200.0000,-970.0000)$

Tool tip coordinates $=\left(\operatorname{tip}_{x}, \operatorname{tip}_{y}\right.$, tip $\left._{z}\right)=(-81.0264,21.2132,-679.4865)$

Normal vector to the plane $\vec{n}=(-78.2625 \hat{\imath}+0 \hat{\jmath}-156.5247 \hat{k})$

Moving platform centre point coordinates $=$ tool tip $-\vec{n}$

$$
(m c p)=\left(m c p_{x}, m c p_{y}, m c p_{z}\right)=(-2.7639, \quad 21.2132, \quad-522.6918)
$$

Analytically, three spherical joints centre point coordinates (Table 2) are determined by (1) and (2).

$$
\begin{aligned}
& S_{1}=(-131.1507,-75.7199,-458.7683) \\
& S_{2}=(127.0333,-73.3427,-587.8606) \\
& S_{3}=(0.0000,194.3907,-524.4338)
\end{aligned}
$$

Table 2. Joint parameters for case study 2(a)

\begin{tabular}{cc}
\hline$\theta$ & Linear actuation \\
\hline$\theta_{1}=60.0586^{\circ}$ & Required linear actuation, $\mathrm{T}_{1}=41.0978 \mathrm{~mm}$ \\
$\theta_{2}=59.4042^{\circ}$ & Required linear actuation, $\mathrm{T}_{2}=172.9648 \mathrm{~mm}$ \\
$\theta_{3}=65.7949^{\circ}$ & Required linear actuation, $\mathrm{T}_{3}=84.7195 \mathrm{~mm}$ \\
\hline
\end{tabular}

Figure 8 shows position and orientation of the tool tip at given points after linear actuation of recirculating ball screw, $T_{1}, T_{2}$ and,$T_{3}$.

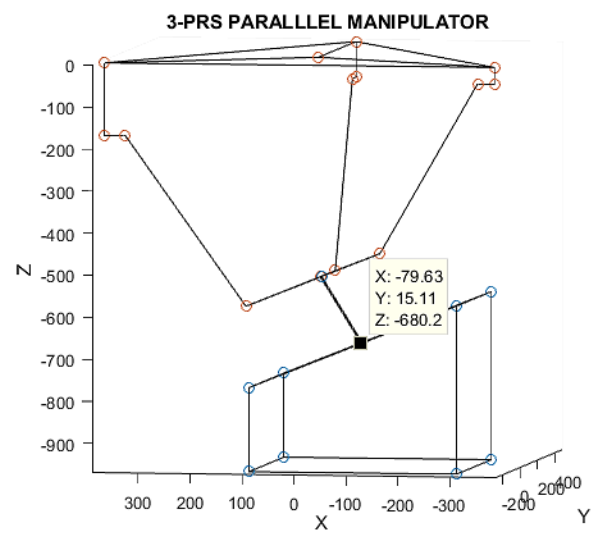

Figure 8. Graphical representation of the position analysis

\section{Case study 2(b)}

Points are selected from the square shape area located on the inclined surface as shown in Figures 9 and 10. Shows position and orientation of the tool tip at given points after linear actuation of recirculating ball screw, $T_{1}, T_{2}$ and,$T_{3}$. Inclination angle of the inclined surface as well as offset distance is same as the previous case. Figure 11 represents the flowchart of position analysis to perform machining operations on an inclined prismatic surface for the 3-PRS manipulator.

\section{Input parameter}

Tool tip coordinates $=(86.5836,0.0000,-676.7079)$

Normal vector to the plane, $\vec{n}=(-78.2625 \hat{\imath}+0 \hat{\jmath}-156.5247 \hat{k})$

Moving platform centre point coordinates $=$ tool tip $-\vec{n}$

$$
m c p=(-2.7639,0.0000,-522.6918)
$$


Analytically, three spherical joints centre point coordinates are determined using (1) and (2).

$$
\begin{aligned}
& S_{1}=(-144.1769,-83.2405,-452.2552) \\
& S_{2}=(131.0382,-75.6549,-589.8631) \\
& S_{3}=(0.0000,172.9550,-524.3438)
\end{aligned}
$$

Note: Here we show position analysis for one point due to page restriction but one can follow same procedure for determining joint parameters for other points which are lies on the same profile. Procedure is shown in the form of flow chart. Table 3 shows results of active and passive joint parameters after inverse kinematic solutions.

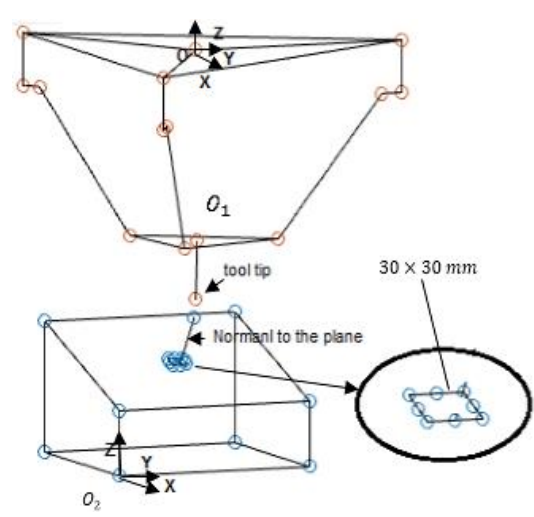

Figure 9. Square shape on inclined surface

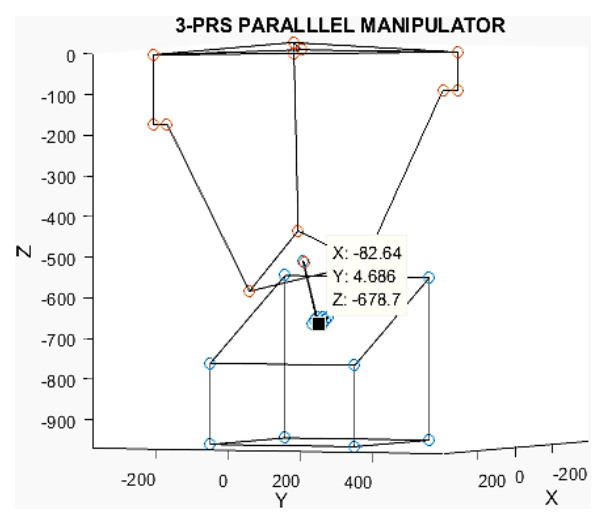

Figure 10. Position analysis on square profile

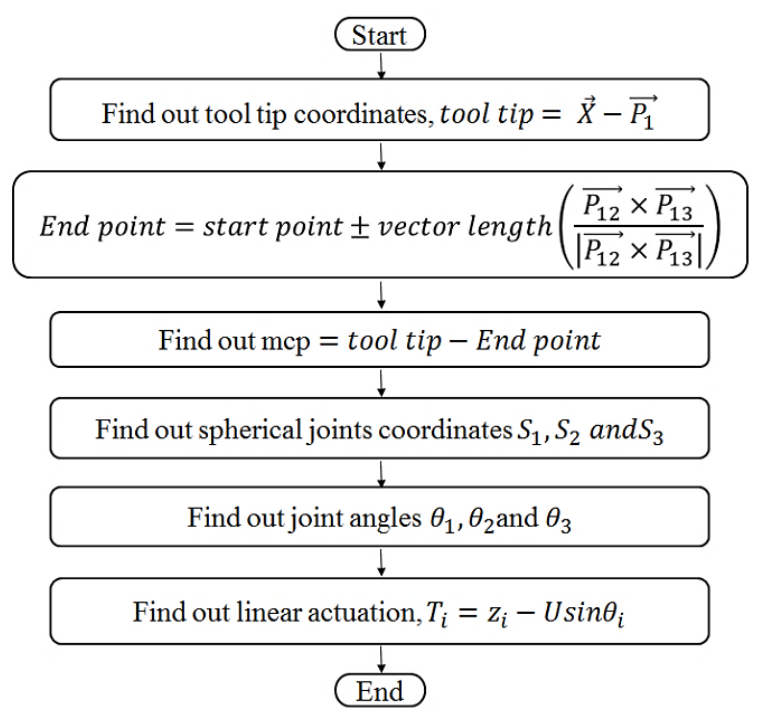

Figure 11. Flow chart for position analysis on inclined surfaces

Table 3. Joint parameters for case study 2(b)

\begin{tabular}{cc}
\hline$\theta$ & Linear actuation \\
\hline$\theta_{1}=62.1014^{\circ}$ & Required linear actuation, , $T_{1}=26.2745 \mathrm{~mm}$ \\
$\theta_{2}=60.0407^{\circ}$ & Required linear actuation, , $T_{2}=172.2674 \mathrm{~mm}$ \\
$\theta_{3}=62.9687^{\circ}$ & Required linear actuation, , $T_{3}=94.9981 \mathrm{~mm}$ \\
\hline
\end{tabular}




\section{CONCLUSION}

Drilling using convention machine is always performed on normal surfaces to predefined machine axis. Holes on inclined surfaces using such machines are feasible using suitable jigs and fixtures with limited accuracy along with proper orientation of job. It is very time consuming and costlier approach. In such circumstances, parallel manipulator could serve this problem within the limited workspace but with high precision and greater consistency. Position analysis of 3-PRS parallel manipulator is carried out using vector approach. Based on inverse kinematic analysis, manipulator is simulated in the MATLAB software as well graphically presented. It is observed that the offset distance is most affective parameter for machining on inclined work part for proposed manipulator. Inverse kinematic solutions are used for machining requirements with tool orientation to produce square and round profiles on inclined surfaces using 3-PRS parallel manipulator.

\section{REFERENCES}

[1] Y. D. Patel, P. M. George, "Parallel Manipulators Applications-A Surve," Modern Mechanical Engineering, vol. 2, no. 03, pp. 57-64, 2012.

[2] Caccavale F., Siciliano B., \& Villani L., "The Tricept robot: dynamics and impedance control," IEEE/ASME transactions on mechatronics, vol. 8, no. 2, pp. 263-268, 2003.

[3] Hennes N., Staimer D., "Application of PKM in aerospace manufacturing-high performance machining centers ECOSPEED, ECOSPEED-F and ECOLINER," In Proceedings of the 4th Chemnitz parallel kinematics seminar, pp. 557-577, 2004.

[4] Y. D. Patel, P. M. George, "Kinematic Analysis and 3D Workspace Development of 3DOF Parallel Manipulator with Retry base," Proceedings of the 1st International and 16th National Conference on Machines and Mechanisms (iNaCoMM2013), IIT Roorkee, India, Dec 18-20 2013

[5] Y. D. Patel, P. M. George, "Constraint and Inverse Kinematic Analysis 3-PRS Parallel Manipulator, 5th International \& 26th All India Manufacturing Technology," Design and Research Conference (AIMTDR 2014) December 12th-14th, 2014.

[6] Pond G., \& Carretero J. A., "Architecture optimisation of three 3-PRS variants for parallel kinematic machining," Robotics and Computer-Integrated Manufacturing, vol. 25, no. 1, pp. 64-72, 2009.

[7] Selvakumar A. A., \& Kumar M. A., "Experimental investigation on position analysis of 3-DOF parallel manipulators," Procedia Engineering, vol. 97, pp. 1126-1134, 2014.

[8] SaioaHerrero, Charles Pinto, Oscar Altuzarra, Mikel Diez, "Analysis of the 2 P RU-1 P RS 3DOF parallel manipulator: kinematics, singularities and dynamics," Robotics and Computer-Integrated Manufacturing, vol. 51, pp. 63-72, 2018

[9] A. Arockia Selvakumar, R. SathishPandian, R. Sivaramakrishnan, K. Kalaichelvan, "Simulation and performance study of 3 - DOF Parallel Manipulator Units," INTERACT, 2010.

[10] Yangmin Li, Qingsong Xu, "Kinematic analysis of a 3-PRS parallel manipulator," Robotics and ComputerIntegrated Manufacturing vol. 23, no. 4, pp. 395-408, 2007.

[11] Pardeshi S. M., \& Doss A. S. A., "Kinematic and Velocity Analysis of 3-DOF Parallel Kinematic Machine for Drilling Operation," Proceedings of the Advances in Robotics, no. 18, pp. 1-6, 2017.

[12] Tsai M. S., Shiau T. N., Tsai Y. J., \& Chang T. H., "Direct kinematic analysis of a 3-PRS parallel mechanism," Mechanism and Machine Theory, vol. 38, no. 1, pp. 71-83, 2003.

[13] Cherfia A., Zaatri A., \& Giordano M., "Kinematics analysis of a parallel robot with a passive segment," Ingeniare Revista chilena de ingeniería, vol. 15, no. 2, pp. 141-148, 2007.

[14] Zhang J., Li Y., \& Huang T., "Dynamic modeling and eigenvalue evaluation of a 3-DOF PKM module," Chinese Journal of Mechanical Engineering, vol. 23, no.2, pp. 1-9, 2010.

[15] Zhang J., Zhao Y. Q., \& Ceccarelli M., "Elastodynamic Model-Based Vibration Characteristics Prediction of a Three Prismatic-Revolute-Spherical Parallel Kinematic Machine," Journal of Dynamic Systems, Measurement, and Control, vol. 138, no. 4, pp. 041009-1-041009-14, 2016. 